\title{
Targeted Lung Deposition From Nebulization Is Not Improved in the Lateral Decubitus Position in Healthy Volunteers
}

\author{
Gregory Reychler, Marta San Miguel-Pagola, Anne-Sophie Aubriot, Beatriz Herrero-Cortina, \\ Vinciane Lecocq, Michel Hesse, Giuseppe Liistro, and François Jamar
}

\begin{abstract}
BACKGROUND: Clinical benefits of inhaled therapies are related to the amount of drug deposited in the targeted area of the lung. Body positions that influence the distribution of lung ventilation should impact lung deposition of the nebulized drug. The aim of this study was to analyze the immediate effect of body position while the subject lies on his side (lateral decubitus) during nebulization on 3-dimensional total and regional lung deposition. METHODS: A randomized crossover trial was performed on healthy male volunteers without cardiovascular or pulmonary disease. A technetium-99m diethylenetriaminepentaacetic acid $\left({ }^{99 \mathrm{~m}}\right.$ Tc-DTPA) solution $(4 \mathrm{~mL})$ was nebulized using an individual-controlled inhalation system in left lateral decubitus and sitting positions with a 96-h washout period (43 inspirations of $4 \mathrm{~s}$ [ie, drug delivered until second 3] with a tidal volume of $0.8 \mathrm{~L}$ and a low inspiratory flow $(200 \mathrm{~mL} / \mathrm{s})$. Drug deposition was followed with the use of planar images and single-photon-emission computed tomography combined with low-resolution computed tomography. Total and regional depositions were the main outcomes. Penetration index was also considered. RESULTS: Six participants $(26.8 \pm 6.9 \mathrm{y}$ old $)$ were included. Total lung deposition tended to be higher in a sitting position than in a lateral decubitus position: $10.2 \pm 0.9 \%$ of nominal dose (95\% CI 9.1-11.3) vs $8.6 \pm 1.4 \%$ of nominal dose (95\% CI 6.8-10.4) $(P=.09)$. The deposition was significantly reduced in the dependent (left) lung in the lateral decubitus position: $3.5 \pm 0.7 \%$ of nominal dose (95\% CI $2.6-4.3)$ vs $4.7 \pm 0.3 \%$ of nominal dose (95\% CI 4.3-5.0) $(P=.03)$. Penetration index was only influenced by body position for the dependent (left) lung $(P=.043)$. CONCLUSIONS: The total amount of drug delivered to the lungs during nebulization with an individual-controlled inhalation system tended to decrease when performed in the left lateral decubitus position. Moreover, contrary to the initial hypothesis, the deposition of particles in the dependent lung was not improved by the lateral decubitus position in this configuration. (ClinicalTrials.gov registration NCT02451501.) Key words: nebulization; aerosol deposition; gamma scintigraphy; body position; delivery; ventilation. [Respir Care 2019;64(12):1537-1544. (C) 2019 Daedalus Enterprises]
\end{abstract}

\section{Introduction}

Inhaled therapy represents the cornerstone of therapeutic strategies in patients suffering from respiratory dis-

Drs Reychler, San Miguel-Pagola, Liistro, and Ms Aubriot are affiliated with Institut de Recherche Expérimentale et Clinique (IREC), Groupe de Recherche en Kinésithérapie Respiratoire, Pôle de Pneumologie, ORL \& Dermatologie, Université Catholique de Louvain, Brussels, Belgium. Drs Reychler, Liistro, and Ms Lecocq are affiliated with Service de Pneumologie, Cliniques Universitaires Saint-Luc, Brussels, Belgium. Dr San Miguel-Pagola and Ms Herrero-Cortina are affiliated with Universidad San Jorge, Campus Universitario Villanueva de Gállego, Zaragoza, Spain. Drs Hesse and Jamar are affiliated with Service de Médecine Nucléaire, Cliniques Universitaires Saint-Luc, Brussels, Belgium. ease. ${ }^{1}$ With the direct delivery of the inhaled drug into the lungs, inhaled therapy has a number of advantages over

\footnotetext{
Dr Reychler has disclosed a relationship with the Institut de Recherche Expérimentale et Clinique (Université catholique de Louvain - Brussels Belgium). The other authors have disclosed no conflicts of interest.

Drs Reychler and San Miguel-Pagola contributed equally to this work.

Correspondence: Gregory Reychler PhD PT, Pneumology Unit, Cliniques Universitaires St-Luc (UCL), Avenue Hippocrate 10, 1200 Brussels, Belgium. E-mail: gregory.reychler@uclouvain.be.
}

DOI: $10.4187 /$ respcare. 06978 
systemic administration such as smaller required dose, faster onset of action, and fewer systemic side effects. ${ }^{2}$ Inhaled therapy is still largely limited to therapies where the lungs are targeted. Clinical benefits are directly correlated with the amount of drug deposited in the targeted area of the lung, ${ }^{3,4}$ which is why modalities of administration need to be optimized, especially when inhaling drugs with a narrow window of efficacy. ${ }^{5}$ The deposition pattern can be influenced by several factors, such as particle characteristics, airway geometry, and breathing pattern. ${ }^{4}$

Body position influences the distribution of lung ventilation, ${ }^{6,7}$ which should impact lung deposition, and the lateral decubitus position (ie, lying on one side) results in the greatest changes with regard to regional ventilation. ${ }^{6,8-11}$ This pattern is due to the effects of gravity, which cause a gradient of pleural pressure attributed to the weight of the lung and a deviation of the mediastinal structures toward one side of the chest cavity, ${ }^{8}$ leading to a decrease in static lung volume. ${ }^{12}$ During spontaneous breathing in adults in either the right or the left lateral decubitus position, ventilation is increased in the less expanded and higher compliance dependent pulmonary area (ie, infralateral lung). ${ }^{6-8,14}$ Body position also influences aerosol pulmonary deposition, even though sitting is the most frequently used position in clinical practice. ${ }^{13,15}$

We hypothesized that nebulization in the lateral decubitus position could help to preferentially target the dependent lung by the modification of ventilation, which would then improve clinical benefits of the nebulized drug. Based on previous research, ${ }^{13}$ this hypothesis should be independent of whether the subject lies on the left or the right side in the lateral decubitus position. The aim of the study was to analyze, in healthy male volunteers, the immediate effect of the left lateral decubitus position during nebulization on 3-dimensional total and regional lung deposition as assessed with single-photon-emission computed tomography combined with low-resolution computed tomography (SPECT-CT).

\section{Methods}

\section{Participants}

Only healthy, nonobese, male volunteer subjects were recruited. Inclusion criteria were age $\geq 18 \mathrm{y}$, never smokers, normal spirometric values, and ability to understand and follow verbal commands. History or evidence of cardiovascular or pulmonary disease, having received any aerosolized drug during the month preceding the experiment, and allergy to technetium-labeled radiopharmaceuticals were exclusion criteria.

The study was performed at Service de Médecine $\mathrm{Nu}$ cléaire, Cliniques Universitaires Saint-Luc, Brussels, Bel-

\section{QUICK LOOK}

\section{Current knowledge}

The efficacy of nebulization depends on the amount of drug deposited in the lungs. The delivery of drug by nebulization in the sitting position is standard practice. Adapting body position could improve targeting of inhaled particles, especially in the lateral decubitus position where ventilation is preferentially distributed to the dependent lung.

\section{What this paper contributes to our knowledge}

The lateral decubitus position decreased the deposition of nebulized particles in the dependent lung. A reduction in total lung deposition was also observed in this position. The lateral decubitus position is therefore not recommended for aerosol delivery.

gium. This study was approved by the Institutional Medical Ethics Committee (BE403201422655). Informed consent was given by all participants prior to the experiment according to the Declaration of Helsinki and current guidelines for good clinical practice.

\section{Design}

This was a single-blind, 2-way, randomized crossover study. Before starting the trial, all subjects performed a selection visit, which included spirometry testing according to the ATS/ERS guidelines ${ }^{16}$ and a medical examination. If the subjects met all of the requirements, they were invited to follow the nebulization training, with a focus on the ability to inhale slowly. Once trained, the subjects performed the first session, consisting of nebulization of the technetium-99m diethylenetriaminepentaacetic $\left({ }^{99 \mathrm{~m}} \mathrm{Tc}\right.$ DTPA) solution using an individual-controlled inhalation system in the corresponding body position (ie, sitting or left lateral decubitus), and the imaging registration procedure (Fig. 1).

After a minimum 96-h washout period, subjects performed the second session, following the same procedure but nebulizing in the other body position studied (Fig. 1). The position order was randomized by an independent investigator using an online computer software (www. randomizer.org) with an allocation ratio of $1: 1$, and the allocation was concealed throughout the entire study. Due to the nature of the intervention, subjects were not blinded to the intervention, whereas the examiners were. The study was performed in accordance with the CONSORT statement for nonpharmacologic trials. 


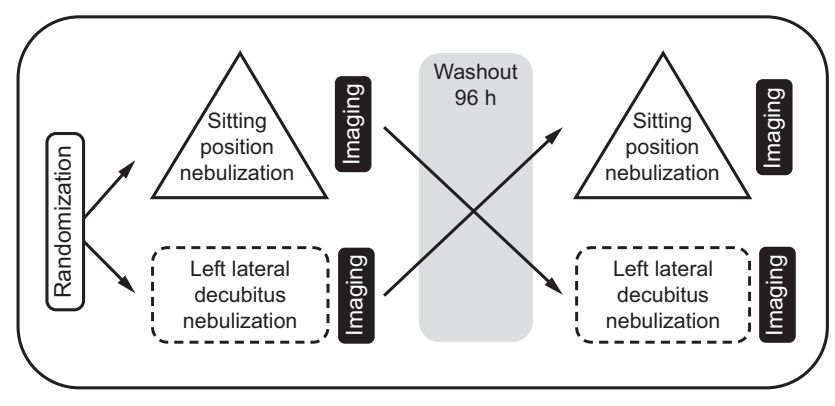

Fig. 1. Schematic of study design.

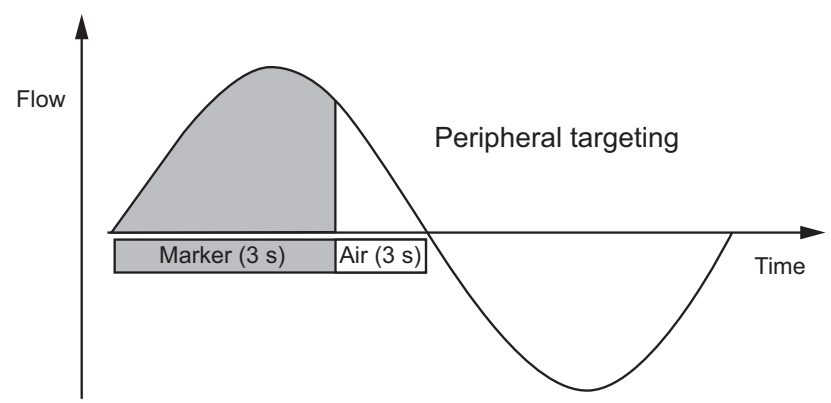

Fig. 2. Illustration of the inhalation waveform and delivery of the nebulized drug.

\section{Nebulization Modalities}

All nebulizations were performed with an individualcontrolled inhalation system (AKITA Jet, Activaero, Gemuenden, Germany), which is a computer-controlled jet or vibrating mesh nebulizer that offers a controlled inhalation and different predetermined programs with controlled regulation of aerosolized drugs during inspiration depending on the targeted site of deposition. In our study, a jet nebulizer (LC Sprint, Pari, Starnberg, Germany) was triggered by the device with an integrated compressor that creates the driving pressure. The mass median aerodynamic diameter (MMAD) was $3.8 \mu \mathrm{m}$.

In this study, a preset targeting peripheral deposition program was used for all nebulizations. Specific settings consisted of 43 inspirations of $4 \mathrm{~s}$ (ie, drug delivered until second 3) with a tidal volume of $0.8 \mathrm{~L}$ and a low inspiratory flow of $200 \mathrm{~mL} / \mathrm{s}$ (Fig. 2). If the inspiratory flow was exceeded, participants received a visual warning on the nebulizer's screen suggesting that they slow down. Although the number of inspirations was arbitrary, these settings assumed an identical delivered amount at the end of the program. During nebulization, subjects were either comfortably seated (sitting position) or in the left lateral decubitus position, and they were asked to breathe through the mouthpiece wearing a nose clip. Subjects were prepositioned in the corresponding study position $15 \mathrm{~min}$ prior to each inhalation to stabilize ventilation distribution. ${ }^{17}$
Both nebulizations were performed randomly at the same time of the day, in the same room, and at ambient temperature.

\section{Nebulization Procedure}

The nebulizer was loaded with $4 \mathrm{~mL}$ of a ${ }^{99 \mathrm{~m}} \mathrm{Tc}$-DTPA solution (TechneScan DTPA, Mallinckrodt Medical, Petten, The Netherlands). The initial activity was measured with a CRC-12 radioisotope calibrator (Capintec, Pittsburgh, Pennsylvania) and was $2.44 \pm 0.17 \mathrm{mCi}$. A one-way valve (19 mL of extra dead-space volume) combined with a filter (Hygrovent, Medisize, The Netherlands) was connected to the nebulizer systems to avoid ambient aerosol contamination and to measure the total activity recovered during expiration. The subjects followed the instructions for slow inhalation given by the device and were supervised by a qualified physiotherapist. Supervision consisted of assuring that subjects were breathing in and out inside the nebulizing circuit without difficulty were and using the correct inspiratory flow. The stop time was determined by the settings of the program. The same nebulization procedure was repeated for both positions.

\section{Imaging Procedure}

Imaging procedures followed the recent standardization of techniques for aerosol-deposition assessment including planar images and SPECT-CT. ${ }^{18}$ A dual-head gamma camera equipped with a low-energy, high-resolution collimator (Philips Brightview XCT, Philips, Milpitas, CA) was calibrated using a point source image without attenuation. The spatial resolution of this system was 3.7 and $7.4 \mathrm{~mm}$ at direct contact and at $10 \mathrm{~cm}$ of the source point, respectively. Images of the device (including nebulizer and filter) and the airways were acquired using a $128 \times 128$ matrix for $2 \mathrm{~min}$. The acquisitions with the flat-panel cone beam CT were performed at $120 \mathrm{kV}$ and $80 \mathrm{~mA}$.

Immediately after nebulization, subjects were invited to lie down on the couch of the gamma camera with their arms positioned above the head. Five sequences were recorded: an anterior planar (AP) image of the filled reservoir of the nebulizer to determine the nominal dose (ie, the radioactivity introduced in the reservoir before the nebulization); a simultaneous $\mathrm{AP}$ and posterior planar (PA) image of the trunk to image aerosol deposition in the stomach and lungs; without moving the subject, a SPECT-CT scan using 60 projection images each of $15 \mathrm{~s}$ and a total imaging time of $8 \mathrm{~min}$; another AP-PA planar image to verify the clearance of the radioactive label and deposition in the upper airways; and an AP planar image of the whole nebulizer after nebulization to quantify aerosol retention. 


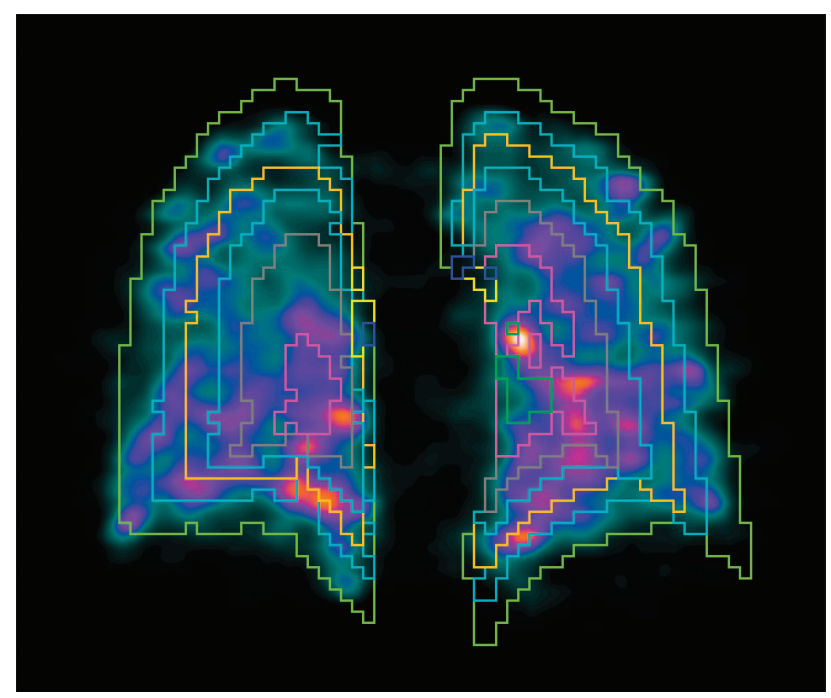

Fig. 3. Shell decomposition of a low-resolution computed tomography coronal slice. Lungs were divided into 10 shells (colored lines through both lungs) distributed from the hilum to the lung periphery. The deposition of the radiolabeled aerosol particles is depicted in color.

\section{Image Analysis}

After reconstruction of SPECT images (Extended Brilliance Workspace, Philips Medical Systems, Best, The Netherlands), images were analyzed (home-made plug-in to ImageJ software, Rasband WS, Bethesda, MD) by a trained technologist who was blinded to the conditions. For the reconstruction, the Astonish iterative algorithm, based on ordered subsets expectation maximization and included attenuation, scatter and resolution recovery corrections, was used. Region of interest and volume of interest were determined, and quantification was performed based on international guidelines. ${ }^{18}$

Volume of interest was derived from the CT, and particles deposited from the trachea to lung periphery were quantified. The trachea and the main bronchi were assembled for the analysis and considered as the tracheal area, and this area was quantified separately from the lungs. Based on international guidelines, ${ }^{18}$ each lung was divided in 10 shells centered on the hilum. Volume of interest was semi-automatically defined. First, trachea, hila, and separation between both lungs were manually positioned based on the CT by visual inspection. The threshold for tissue differentiation was fixed by the assessor at approximately -400 and -750 Hounsfield units for lung and trachea determination, respectively. Second, shells and volumes of interest were automatically created.

Deposition in each lung was calculated by the total lung cumulated counts obtained by summing the counts measured in the 10 shells of the corresponding lung (Fig. 3). Total lung deposition was calculated by summing the total lung cumulated counts from both lungs. The right/left lung ratio was calculated. Trachea deposition was derived from the corresponding volume of interest. Penetration index was calculated as the outer/inner zone radioactivity ratio from the SPECT scan normalized by the same ratio for ventilation zones from the $\mathrm{CT}$ scan. The outer region corresponds to the 5 distal shells, and the 5 proximal shells determine the inner region.

A rectangle was drawn on AP-PA images to quantify lung deposition and initial and residual radioactivity in the device. Mass balance was determined from planar images by comparing the radioactivity initially placed in the nebulizer reservoir and expressed in counts against the radioactivity recovered in all locations after nebulization. The mean mass balance was checked to be $100 \% \pm 10 \% .{ }^{19}$

The doses were expressed as percentage of the nominal dose of radioactivity. Quantifications were corrected for decay of radioactivity with a time correction factor (ie, using the time between the acquisition of the filled nebulizer and each image deposition), for background (ie, using a region outside of the radiolabeled area), and with an attenuation correction factor (ie, using thorax thickness from $\mathrm{CT}$ ) based on international guidelines. ${ }^{18}$

\section{Statistical Analysis}

To detect a minimal difference of $10 \%$ of total lung deposition during nebulization between the 2 sessions, accepting an alpha risk of 0.05 and a beta risk of 0.2 in a 2 -sided test, and assuming that $6 \%$ is the standard deviation of the difference in the response to treatment for the same participant, ${ }^{20} 6$ subjects were necessary.

After verifying the normality of the distribution by Kolmogorov-Smirnov test, results were expressed as mean \pm SD with $95 \%$ CI. Statistical tests were performed using SPSS Statistics 23.0 (IBM, Armonk, NY). The paired Student $t$ test was used for means comparisons of parameters related to deposition between the 2 positions. The initial radioactivity of the reservoirs and the activity retained in the nebulizer at the end of the nebulization was compared using an unpaired Student $t$ test.

\section{Results}

Six healthy nonobese males were enrolled and randomized in the study, and all 6 subjects completed the study (Fig. 4). Table 1 shows the anthropometric and cardiorespiratory parameters of the subjects measured at baseline. Lung function was in the normal range for all subjects.

The initial load in the nebulizer was similar in both positions $(P=.81)$. No ambient or surface contamination was detected after nebulization. The residual radioactivity in the nebulizer was similar in both positions: $82.6 \pm 2.9 \%$ and $81.7 \pm 3.8 \%$ of nominal dose for sitting and lateral 


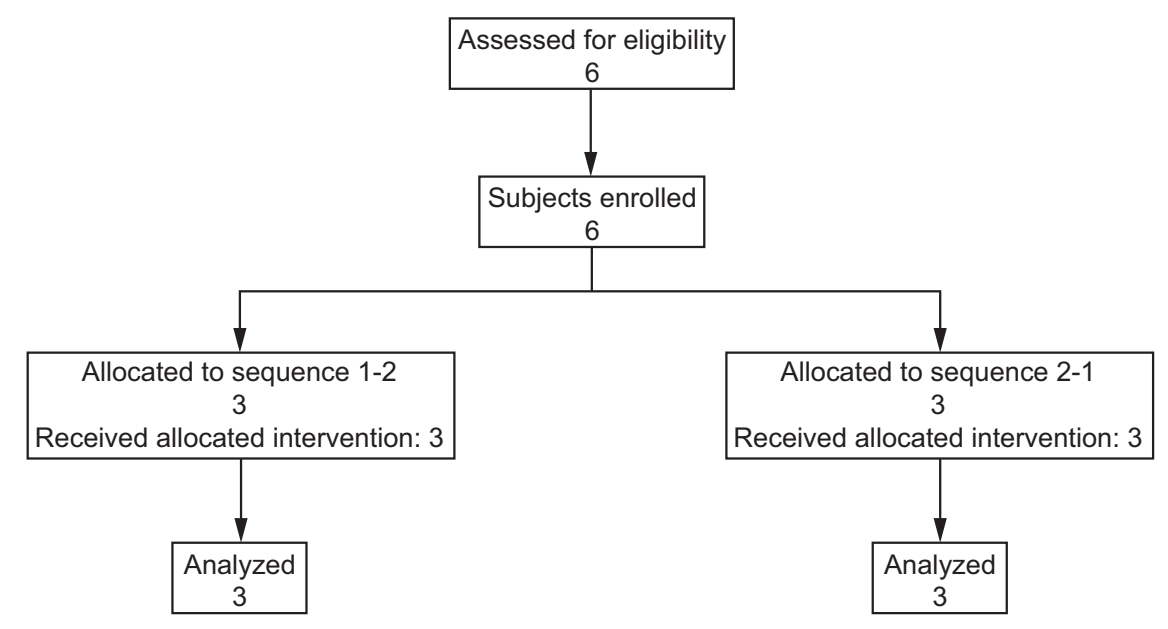

Fig. 4. Flow chart.

Table 1. Subject Characteristics and Lung Function Parameters

\begin{tabular}{lc}
\hline \hline & Mean \pm SD \\
\hline Age, $y$ & $26.8 \pm 6.9$ \\
Height, cm & $182.5 \pm 9.9$ \\
Weight, $\mathrm{kg}$ & $77.5 \pm 11.3$ \\
Body mass index, $\mathrm{kg} / \mathrm{m}^{2}$ & $23.2 \pm 2.6$ \\
FEV $_{1} / \mathrm{FVC}, \%$ & $87.0 \pm 6.2$ \\
FEV $_{1} \%$ of predicted & $89.5 \pm 8.0$ \\
FVC $\%$ of predicted & $96.3 \pm 10.9$ \\
\hline
\end{tabular}

positions, respectively $(P=.65)$. The results of deposition with the 2 procedures are represented in Table 2 . The total lung deposition tended to be lower in the lateral position than in the sitting position, with a mean difference of $-1.6 \%$ (95\% CI -3.5 to $0.4, P=.09$ ), without modifying the tracheal deposition $(P=.61)$, and intersubject variability was twice as great in this position (coefficient of variation: $16.3 \%$ lateral decubitus vs $8.9 \%$ sitting). Whereas the left lateral decubitus position had little effect on deposited dose in the right lung compared to the sitting position (5.1\% vs $5.5 \%$ of nominal dose, $P=.45$ ), it significantly reduced deposition in the left (dependent) lung (3.5\% vs $4.7 \%$ of nominal dose, $P=.03$ ). There is a tendency to a greater left/right lung deposition ratio in the sitting position than in the lateral decubitus position. Deposition was greater in the right lung compared to the left lung for all subjects in both positions as illustrated by the left/right lung deposition ratio $<100 \%$ for all participants (Fig. 5). All but one subject had a higher ratio in the sitting position than in the lateral decubitus position. Penetration index was only influenced by the position for the dependent (left) lung $(P=.043)$. The extrathoracic deposition (excluding tracheal area) was $10.1 \pm 3.1 \%$ and $7.2 \pm 3.6 \%$ of nominal dose for the lateral decubitus position and the sitting position, respectively $(P=.051)$.

\section{Discussion}

This randomized, controlled study highlighted that nebulization with a controlled-dose, breath-actuated nebulizer system in the lateral decubitus position was associated with lower total lung deposition secondary to reduced deposition in the dependent lung in healthy volunteers. In addition, a lower proportion of the nominal aerosolized dose tended to reach all regions of the lungs in this position. The clinical relevance of these findings is that the lateral decubitus position does not improve total or targeted lung delivery. Our results also confirm the low variability in deposition when nebulizing with a controlleddose inhalation drug-delivery system (ie, AKITA Jet). ${ }^{20,21}$

A difference in total lung deposition related to body position was previously suggested and explained by an increased extrathoracic deposition, but this observation was only based on the results for one subject. ${ }^{22}$ No difference in tracheal and extrathoracic deposition was observed in our study, although extrathoracic deposition probably contributed to the reduced total lungs deposition in the left lateral decubitus position. Independent of the total amount of drug reaching the lungs, the deposited amount of nebulized drug was higher in the right lung than in the left lung. This is well known to occur in an erect position, and it is related to the greater static volume and better ventilation of the right lung. ${ }^{23}$ However, contrary to our initial hypothesis and to the results of a previous study evaluating aerosol lung deposition in the 2 lateral decubitus positions, ${ }^{13}$ even though the left lateral decubitus position modified the distribution of particle deposition between both lungs, this modality of administration did not improve deposition in the dependent lung. The fact that position and distribution of ventilation do not affect the distribution of the deposited particles in healthy subjects has been reported previously, although the uniformity of the distri- 
Table 2. SPECT-CT Analysis of Aerosol Deposition

\begin{tabular}{|c|c|c|c|c|}
\hline & Left Lateral Decubitus & Sitting & Mean Difference & $P$ \\
\hline Both lungs, \% ND & $8.6 \pm 1.4(6.8-10.4)$ & $10.2 \pm 0.9(9.1-11.3)$ & -1.6 to $\pm 1.6(-3.5-0.3)$ & .09 \\
\hline Right lung, \% ND & $5.1 \pm 0.8(4.1-6.1)$ & $5.5 \pm 0.8(4.5-6.5)$ & $-0.4 \pm 1.1(-1.7$ to 0.9$)$ & .45 \\
\hline Left lung, \% ND & $3.5 \pm 0.7(2.6-4.3)$ & $4.7 \pm 0.3(4.3-5.0)$ & $-1.2 \pm 0.8(-2.2$ to -0.2$)$ & .03 \\
\hline Left/right lung ratio & $0.68 \pm 0.08(0.58-0.77)$ & $0.86 \pm 0.10(0.73-0.98)$ & $-0.18 \pm 0.16(-0.37$ to 0.01$)$ & .061 \\
\hline \multicolumn{5}{|l|}{ Penetration index } \\
\hline Right lung & $0.63 \pm 0.15(0.44-0.82)$ & $0.65 \pm 0.23(0.36-0.94)$ & $-0.02 \pm 0.19(-0.26$ to 0.22$)$ & .93 \\
\hline Left lung & $0.46 \pm 0.11(0.33-0.59)$ & $0.61 \pm 0.12(0.47-0.76)$ & $-0.16 \pm 0.11(-0.29$ to -0.02$)$ & .043 \\
\hline Tracheal area, \% ND & $0.48 \pm 0.41(0.02-0.99)$ & $0.45 \pm 0.21(0.18-0.72)$ & $-0.8 \pm 2.2(-3.2$ to 1.5$)$ & .61 \\
\hline
\end{tabular}

Data expressed as mean \pm SD $(95 \% \mathrm{CI})$

SPECT-CT $=$ single photon emission computed tomography combined with computed tomography

$\% \mathrm{ND}=$ percentage of nominal dose

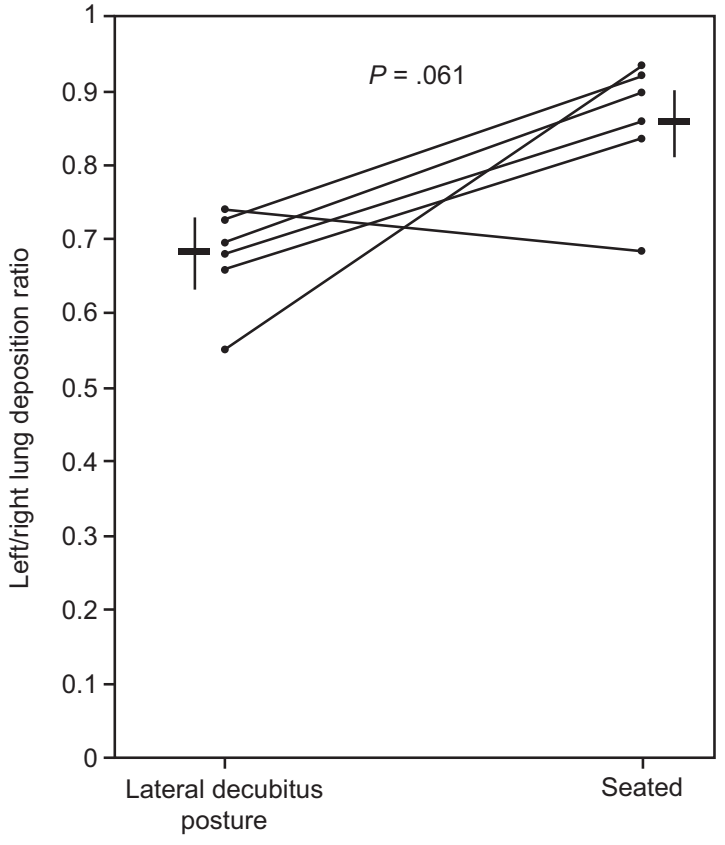

Fig. 5. Left/right lung deposition ratio in both positions.

bution could have been increased due to the fine size of nebulized particles (MMAD $2 \mu \mathrm{m}$ ). ${ }^{24}$ Indeed, it is well known that particle size plays an important role in lung deposition. ${ }^{25}$

Moreover, the left/right lung ratio was reduced in the left lateral decubitus position, which means that the proportion of drug deposited in the right lung (nondependent lung) was greater in the left lateral decubitus position than in the sitting position, even though the total amount deposited in this lung was reduced by the lateral decubitus position. Surprisingly, in our study, the lateral decubitus position preferentially targeted the nondependent lung. Different elements can contribute to the lower-than-expected deposition in the left lung. The reduced static volume of the dependent lung in this position as mentioned above ${ }^{26}$ and the potential micro-atelectasis generated by the position at end expiration could reduce the delivered drug in this lung. The expiratory filter included in our device could play a role in our results by generating a positive expiratory pressure. Indeed, a shift neutralization of the spatial distribution toward the dependent lung in the lateral decubitus position during spontaneous breathing through a positive expiratory pressure device was demonstrated in healthy subjects. ${ }^{27}$ Thus, the resistance generated by the expiratory filter, even if lower than a classical positive expiratory pressure device, hypothetically provided a more homogenous ventilation distribution between both lungs.

The nebulizer settings of the individual-controlled inhalation system (AKITA Jet) from our study versus a jet nebulizer (Venticis II, CIS Biointemational, France) could also be responsible for the differences. The Venticis II jet nebulizer is intended for the study of pulmonary ventilation or alveolar-capillary permeability using radiopharmaceutical products labeled with $99 \mathrm{~m}$-technetium. Because the drug was inhaled slowly with a large tidal volume and particles were only administered at the beginning of inspiration with this device, it is possible that the drug had more difficulty reaching some parts of the dependent lung. Indeed, the filling characteristics or the rate of volume change of the dependent lung during the initial part of inspiration was reduced by greater tidal volumes ${ }^{11}$ and to be lower than the nondependent lung. ${ }^{26}$ This means that the dependent lung expanded more slowly than the nondependent lung in this condition. Moreover, the MMAD of the aerosol generated by the individual-controlled inhalation system was greater $(3.8 \mu \mathrm{m})$ than that produced by the Venticis II jet nebulizer $(0.9 \mu \mathrm{m}$ under 1.5 bar pressure and at a flow of $25.1 \mathrm{~L} / \mathrm{min}$ ), and particle size is directly related to lung deposition. ${ }^{25}$

The distribution of the deposited particles inside each lung is also influenced differently by the subject's position. The deposition in the dependent lung was less peripheral with a lower penetration index in the lateral de- 
cubitus position than in the sitting position. It can be similarly explained by the nebulizer settings. Indeed, the chosen modality of administration delivered the drug during the first part of inspiration. The slower expansion of the dependent lung during the beginning of inspiration in the lateral decubitus position combined with this method of administration was unfavorable to peripheral deposition in this lung. Moreover, the reduced airway diameter in the dependent lung when the subject was laying on one side increased the inertial impaction and thus the deposition in its central zones resulting from the higher flow generated in this lung. ${ }^{28} \mathrm{~A}$ similar result was reported by Sá et al ${ }^{15}$ in the supine position. They showed decreased deposition in the alveolar region when inhalation occurred in the supine position compared to a sitting position. On the other hand, body position did not influence the distribution of deposition in the nondependent region of the lung because the penetration index from this lung was similar in both positions. However, the variability of the penetration index in the nondependent region of the lung was $50 \%$ higher in the sitting position.

From a clinical perspective, when using a nebulizer promoting the delivery of particles during the first part of inspiration, the lateral decubitus position may not be beneficial to total lung deposition because this position tends to reduce total delivery to the lungs. Moreover, the drug targeting of the dependent lung by the lateral decubitus position was not verified in our results, suggesting that this modality of nebulization does not improve the delivery of particles in this lung.

One of the strengths of this study was the similar pattern of breathing for all subjects during nebulization in the 2 positions. Indeed, the individual-controlled inhalation system is a specific nebulizer guiding the user's pattern of breathing. This ensures a reproducible pattern of breathing (particularly the inspiratory volume and duration), eliminates its influence on lung deposition, ${ }^{29}$ and thus reduces inter-patient variability of total and peripheral deposition. ${ }^{21}$ The variability of lung and peripheral deposition is 4 times lower when breathing is guided by the individual-controlled inhalation system in comparison to a classic nebulizer. ${ }^{21}$ Moreover, inspiratory flow was standardized by the nebulizer to reduce its influence on the comparison. Indeed, the inspiratory period highly influences lung deposition and determines deposition variability. ${ }^{30}$

Our study has some limitations. The delivered dose seemed low for the individual-controlled inhalation system, but this was related to the lower preset number of inhalations chosen in our study. Because we chose healthy male subjects, the generalizability to patients could be an issue. Indeed, patients can present heterogeneously with regard to ventilation, secretions, reduced airway diameter, and lung compliance, which could result in different findings. Our study, however, was based on physiological pa- rameters and responded thus to physiological questioning. In spite of this, it is usual when evaluating a nebulization modality to first study healthy subjects to reduce the influence of poorly controlled disease on lung deposition. Indeed, nebulized particles are more centrally deposited, and a wide range of distribution between central and peripheral zones is observed in patients with obstructive lung disease. Moreover, Brand et al ${ }^{31}$ reported that the individual-controlled inhalation system reduced disease influence on lung deposition, particularly on the central/peripheral deposition ratio. In our study, we only recruited male subjects; however, sex would probably not be consequential to the study due to the paired design and the poor influence of gender specificity toward attenuation factors. Although a small but statistically sufficient number of subjects was investigated, the group was homogeneous in terms of lung function, which is probably the main influencing parameter on lung deposition in healthy subjects. The study was underpowered to detect differences smaller than the 10 percentage point difference anticipated due to body position, which can be considered as a clinically important characteristic. Finally, the particle size of the nebulizer was not measured with the nebulized solution. Our data are based on the determination from a sodium fluoride solution $(3 \mathrm{~mL}-2.5 \%)$ at a flow of $15 \mathrm{~L} / \mathrm{min}$.

\section{Conclusions}

The lateral decubitus position reduced the total amount of drug delivered to the lungs when nebulization was performed in specified settings with the individual-controlled inhalation system in healthy male volunteers. In addition, the deposition is less distal in the dependent lung. Moreover, a lower proportion of the nominal dose reached all regions of the lungs in this position, especially the dependent lungs. Contrary to the initial hypothesis, the deposition of particles in the dependent lung is not improved by the lateral decubitus position in this configuration. Our findings do not support the routine use of the lateral decubitus position during nebulization when using the individual-controlled inhalation system. Clinically, the lateral decubitus position is not recommended to target a specific pulmonary area because it may negatively affect the clinical benefit of the nebulized drug due to the lower delivered dose.

\section{ACKNOWLEDGMENT}

We thank Jonathan Dugernier for his help with the reconstruction of the images.

\section{REFERENCES}

1. Roche N, Scheuch G, Pritchard JN, Nopitsch-Mai C, Lakhani DA, Saluja B, et al. Patient focus and regulatory considerations for inha- 


\section{Targeted Lung Deposition in Lateral Decubitus Position}

lation device design: Report from the 2015 IPAC-RS/ISAM Workshop. J Aerosol Med Pulm Drug Deliv 2017;30(1):1-13.

2. Capstick TG, Clifton IJ. Inhaler technique and training in people with chronic obstructive pulmonary disease and asthma. Expert Rev Respir Med 2012;6(1):91-101.

3. Newman SP. Can lung deposition data act as a surrogate for the clinical response to inhaled asthma drugs? Br J Clin Pharmacol 2000;49(6):529-537.

4. Darquenne C. Aerosol deposition in health and disease. J Aerosol Med Pulm Drug Deliv 2012;25(3):140-147.

5. Laube BL. The expanding role of aerosols in systemic drug delivery, gene therapy, and vaccination. Respir Care 2005;50(9):1161-1176.

6. Ericsson E, Tesselaar E, Sjoberg F. Effect of electrode belt and body positions on regional pulmonary ventilation- and perfusion-related impedance changes measured by electric impedance tomography. PLoS One 2016;11(6):e0155913.

7. Frerichs I, Braun P, Dudykevych T, Hahn G, Genee D, Hellige G. Distribution of ventilation in young and elderly adults determined by electrical impedance tomography. Respir Physiol Neurobiol 2004; 143(1):63-75.

8. Grant CA, Pham T, Hough J, Riedel T, Stocker C, Schibler A. Measurement of ventilation and cardiac related impedance changes with electrical impedance tomography. Crit Care 2011;15(1):R37.

9. Kaneko K, Milic-Emili J, Dolovich MB, Dawson A, Bates DV. Regional distribution of ventilation and perfusion as a function of body position. J Appl Physiol 1966;21(3):767-777.

10. Krieg S, Alison JA, McCarren B, Cowell S. Position affects distribution of ventilation in the lungs of older people: an experimental study. Aust J Physiother 2007;53(3):179-184.

11. Schnidrig S, Casaulta C, Schibler A, Riedel T. Influence of endexpiratory level and tidal volume on gravitational ventilation distribution during tidal breathing in healthy adults. Eur J Appl Physiol 2013;113(3):591-598.

12. Elliott AR, Prisk GK, Guy HJ, West JB. Lung volumes during sustained microgravity on Spacelab SLS-1. J Appl Physiol (1985) 1994; 77(4):2005-2014.

13. Alcoforado L, Pessoa Filho LC, Brandao DC, Galvao AM, Reinaux $\mathrm{CM}$, Andrade $\mathrm{AD}$. Influence of change in lateral decubitus on pulmonary aerosol deposition. Rev Bras Fisioter 2011;15(4):278-283.

14. West JB, Dollery CT. Distribution of blood flow and ventilationperfusion ratio in the lung, measured with radioactive carbon dioxide. J Appl Physiol 1960;15:405-410.

15. Sá RC, Zeman KL, Bennett WD, Prisk GK, Darquenne C. Effect of posture on regional deposition of coarse particles in the healthy human lung. J Aerosol Med Pulm Drug Deliv 2015;28(6):423-431.

16. Miller MR, Hankinson J, Brusasco V, Burgos F, Casaburi R, Coates A, et al. Standardisation of spirometry. Eur Respir J 2005;26(2):319338.

17. Caruana L, Paratz JD, Chang A, Barnett AG, Fraser JF. The time taken for the regional distribution of ventilation to stabilise: an investigation using electrical impedance tomography. Anaesth Intensive Care 2015;43(1):88-91.
18. Fleming J, Bailey DL, Chan HK, Conway J, Kuehl PJ, Laube BL, et al. Standardization of techniques for using single-photon emission computed tomography (SPECT) for aerosol deposition assessment of orally inhaled products. J Aerosol Med Pulm Drug Deliv 2012;25 Suppl 1:S29-S51.

19. Newman S, Bennett WD, Biddiscombe M, Devadason SG, Dolovich $\mathrm{MB}$, Fleming J, et al. Standardization of techniques for using planar (2D) imaging for aerosol deposition assessment of orally inhaled products. J Aerosol Med Pulm Drug Deliv 2012;25(Suppl 1):S10S28.

20. Reychler G, Aubriot AS, Depoortere V, Jamar F, Liistro G. Effect of drug targeting nebulization on lung deposition: a randomized crossover scintigraphic comparison between central and peripheral delivery. Respir Care 2014;59(10):1501-1507.

21. Brand P, Beckmann H, Maas EM, Meyer T, Mullinger B, Sommerer $\mathrm{K}$, et al. Peripheral deposition of alpha1-protease inhibitor using commercial inhalation devices. Eur Respir J 2003;22(2):263-267.

22. Conway J, Fleming J, Majoral C, Katz I, Perchet D, Peebles Ch, et al. Controlled, parametric, individualized, 2-D and 3-D imaging measurements of aerosol deposition in the respiratory tract of healthy human subjects for model validation. J Aero Sci 2012;52:1-17.

23. Majoral C, Fleming J, Conway J, Katz I, Tossici-Bolt L, Pichelin M, et al. Controlled, parametric, individualized, 2D and 3D imaging measurements of aerosol deposition in the respiratory tract of healthy human volunteers: in vivo data analysis. J Aerosol Med Pulm Drug Deliv 2014;27(5):349-362

24. Chan HK, Daviskas E, Eberl S, Robinson M, Bautovich G, Young I. Deposition of aqueous aerosol of technetium-99m diethylene triamine penta-acetic acid generated and delivered by a novel system (AERx) in healthy subjects. Eur J Nucl Med 1999;26(4):320-327.

25. Heyder J. Deposition of inhaled particles in the human respiratory tract and consequences for regional targeting in respiratory drug delivery. Proc Am Thorac Soc 2004;1(4):315-320.

26. Milic-Emili J, Henderson JA, Dolovich MB, Trop D, Kaneko K. Regional distribution of inspired gas in the lung. J Appl Physiol 1966;21(3):749-759.

27. Wettstein M, Radlinger L, Riedel T. Effect of different breathing aids on ventilation distribution in adults with cystic fibrosis. PLoS One 2014;9(9):e106591.

28. Scheuch G, Stahlhofen W. Particle deposition of inhaled aerosol boluses in the upper human airways. J Aerosol Sci 1987;18(6):725727.

29. Bennett WD, Smaldone GC. Human variation in the peripheral airspace deposition of inhaled particles. J Appl Physiol 1987;62(4): 1603-1610

30. Bennett WD, Zeman KL, Kim C. Variability of fine particle deposition in healthy adults: effect of age and gender. Am J Respir Crit Care Med 1996;153(5):1641-1647.

31. Brand P, Schulte M, Wencker M, Herpich CH, Klein G, Hanna K, et al. Lung deposition of inhaled alpha1-proteinase inhibitor in cystic fibrosis and alpha1-antitrypsin deficiency. Eur Respir J 2009;34(2): 354-360. 